\title{
Molecular mechanism of hepatocellular carcinoma-specific antitumor activity of the novel thienopyridine derivative TP58
}

\author{
RUI ZHOU ${ }^{1,2 *}$, WEN-JUN HUANG ${ }^{2 *}$, ZHI-YUN GUO ${ }^{3 *}$, LEI LI $^{4}$, XIAO-RONG ZENG ${ }^{2}$, \\ YA-QI DENG ${ }^{1}$, FENG-YUN HU ${ }^{1}$, AI-PING TONG ${ }^{1}$, LI YANG $^{1}$ and JIN-LIANG YANG ${ }^{1}$
}

\author{
${ }^{1}$ State Key Laboratory of Biotherapy and Cancer Center, West China Hospital, West China Medical School, \\ Sichuan University, Chengdu 610041; ${ }^{2}$ Department of Electrophysiology, Institute of Cardiovasology, Luzhou \\ Medical College, Luzhou, Sichuan 646000; ${ }^{3}$ School of Life Science and Engineering, Southwest Jiaotong University, \\ Chengdu 10031; ${ }^{4}$ Department of Radiation Oncology, Shanxi Provincial People's Hospital, Taiyuan 030000, P.R. China
}

Received January 17, 2012; Accepted March 5, 2012

DOI: 10.3892/or.2012.1776

\begin{abstract}
Despite progress made in the treatment of hepatocellular carcinoma (HCC), there is no curable treatment. Novel therapies are therefore needed. In our previous study on the design and synthesis of a small molecular inhibitor targeting Aurora kinase, we discovered a novel thienopyridine derivative compound (1g, TP58) which displayed the most potent and relatively specific inhibition of the proliferation of HepG2 human hepatoma cells in vitro. However, the molecular mechanism remains to be elucidated. Herein, in vitro and in vivo studies were conducted to further verify its antitumor activity against HCC. cDNA microarray and two-dimensional protein gel electrophoresis technology were utilized to elucidate the mechanism of HCC-specific inhibition of TP58. Flow cytometry analysis displayed that TP58 can significantly induce G0/ G1 arrest in HepG2 cells. Sixteen genes involved in cell cycle regulation were found to be dysregulated following TP58 treatment using microarray technology. Nine proteins whose expression was altered (corresponding to 10 spots identified as differentially expressed) were identified by proteomic analysis. Further study showed that TP58 can modulate the expression of some liver-enriched transcription factors (LETFs) and liverspecific marker genes, such as hepatic nuclear factor (HNF-4)
\end{abstract}

Correspondence to: Dr Ai-Ping Tong or Dr Li Yang, State Key Laboratory of Biotherapy and Cancer Center, West China Hospital, West China Medical School, Sichuan University, Chengdu 610041, P.R. China

E-mail: aipingtong@scu.edu.cn

E-mail: yangli@scu.edu.cn

*Contributed equally

Abbreviations: HCC, hepatocellular carcinoma; cDNA, microarray; LETFs, liver-enriched transcription factors; HNF, hepatic nuclear factor

Key words: hepatocellular carcinoma, cDNA microarray, proteomics, liver-enriched transcription factors and $\alpha$-fetoprotein (AFP). These findings may help explain the mechanism of HCC-specific antitumor activity of TP58 and provide some useful insight for anti-HCC drug design and future use of thienopyridine derivatives in HCC therapy.

\section{Introduction}

Hepatocellular carcinoma (HCC), a major form of malignancy of liver with a worldwide increasing incidence, is one of the most common causes of cancer-related deaths in the world (1). The potential curative therapeutic options are surgical procedures, such as liver transplantation and surgical resection. Unfortunately, the majority $(>80 \%)$ of the patients with advanced and unresectable HCC are not eligible for these techniques (2). Chemotherapy is one of the most commonly used treatment option, especially for patients with unresectable tumors. However, the use of conventional cytotoxic drugs has shown no improvement in survival, and patients suffer some adverse effects (3). Therefore, development of novel adjuvant therapies targeting liver cancer growth is essential.

The Aurora kinase, a family of highly conserved serinethreonine kinases, consisting of three isoforms (Aurora A, Aurora B and Aurora C), have integral functions in mitotic cell division, tumor development and prognosis $(4,5)$. Overexpression and amplification of Aurora A in HCC have been found to be correlated with high grade and high stage tumors (6). It has been reported that Aurora B is not only associated with hepatocellular carcinoma (HCC) recurrence (7) but with independent molecular marker predicting tumor invasiveness and poor prognosis of HCC (8). More recent studies have shown that the administration of small molecule inhibitors of Aurora kinases resulted in significant tumor growth inhibition through inducing proliferation blockade, histone H3 (Ser10) dephosphorylation and apoptosis (9-11). All these suggest that Aurora kinases may be potential candidates for novel therapies for liver tumor growth, recurrence and metastasis.

In our previous study, a series of analogs were designed and synthesized based on a hit thienopyridine compound (1a, TP01) targeting Aurora kinases by a restricted de novo design strategy (12). Among these analogs, TP58 was the most 
potent $\left(\mathrm{IC}_{50}=0.016 \mu \mathrm{M}\right.$ vs. $\mathrm{IC}_{50}=0.37 \mu \mathrm{M}$ for doxorubicin as a positive control) and relatively specific $\left(\mathrm{IC}_{50}>100 \mu \mathrm{M}\right.$ in five different types of human cancer cell lines) in terms of the inhibition of proliferation of the human hepatoma HepG2 cells in vitro. The precise mechanism of the TP58 effects has not been fully elucidated. In our current study, we investigated the potential anticancer effect of TP58 on hepatocellular carcinoma through in vitro function studies and a liver tumor animal model in vivo. The combined application of two high throughput methods, cDNA microarray and two-dimensional protein gel electrophoresis technology provide us with suffice amounts of data at both the transcriptional and translational levels, respectively, thus providing a better understanding of the mechanism of HCC-specific inhibition. Finally, western blot analysis was performed to determined members of liverenriched transcription factors (LETFs) including hepatic nuclear factor 1 (HNF-1), hepatic nuclear factor 3 (HNF-3), hepatic nuclear factor 4 (HNF-4), and CCAAT/enhancer binding protein (C/EBP), which play a crucial role in the development and differentiation of liver through relative restricted expression in liver and regulating transcription of liver specific genes, such as AFP $(13,14)$. These findings may help explain the mechanisms of HCC-specific antitumor activity of TP58 and provide some useful clues for anti-HCC drug design and future use of thienopyridine derivatives in HCC therapy.

\section{Materials and methods}

HepG2 cell line. The human hepatocellular carcinoma cell line HepG2 and the normal human liver cell line L02 was obtained from the American Type Culture Collection (ATCC, Rockville, MD, USA) and was cultured according to ATCC recommendations. Cells were maintained in Dulbecco's modified Eagle medium (DMEM) supplemented with 10\% FBS (Gibco, Eggenstein, Germany) at $37^{\circ} \mathrm{C}$ under an atmosphere of $95 \% \mathrm{O}_{2}, 5 \% \mathrm{CO}_{2}$.

Reagent and antibodies. For in vitro assays, TP58 was dissolved initially as a $20 \mathrm{mM}$ stock solution in dimethylsulfoxide (DMSO). The stock solution was diluted to $1 \mu \mathrm{M}$ in the relevant assay media and $0.1 \%$ DMSO served as a vehicle control. For in vivo studies, TP58 was dissolved in $1 \%$ polyethylene glycol solution containing $2 \%$ glycerol. Phosphorylated HSP 27 (Ser82) (p-HSP) antibody was obtained from Abcam. Hepatic nuclear factor $1 \alpha$ (HNF-1 $\alpha$ ), hepatic nuclear factor $3 \beta$ (HNF-3 $\beta$ ), hepatic nuclear factor $4 \alpha$ (HNF- $4 \alpha)$, and C/EBP $\alpha$ antibodies were obtained from Epitomics. AFP and $\beta$-actin antibodies were obtained from Santa Cruz Biotechnology. Horseradish peroxidase (HRP)-conjugated anti-mouse IgG and HRP-conjugated anti-rabbit IgG were obtained from Zhongshan Golden Bridge (Beijing, China).

Proliferation assay. Cell proliferation was measured using the MTT assay as previously described (15). Cells were treated with indicated concentrations of TP58 for $36 \mathrm{~h}$. Each assay was performed in 5 replicates.

Cell cycle analysis. Cells were harvested by trypsinization $36 \mathrm{~h}$ after treatment, washed three times with ice-cold PBS, and fixed with $70 \%$ ethanol overnight at $4^{\circ} \mathrm{C}$. The fixed cells were rehydrated in PBS, and subjected to PI/RNase staining followed by FACS (fluorescence activated cell sorter scan) analysis (Becton Dickinson, Mountain View, CA, USA). The percentage of cells in each phase of the cell cycle was estimated using Elite software.

Apoptosis analysis. Nuclear morphology of control and TP58-treated cells was observed by staining cell nuclei with Hoechst 33342 (Sigma, St. Louis, MO, USA). Cells (at least 200 per slide) were incubated with Hoechst $33342(10 \mu \mathrm{g} / \mathrm{ml})$ for $15 \mathrm{~min}$ at RT and examined under a fluorescence microscope (Olympus).

Western blot analysis. Total-cell lysates were prepared using RIPA buffer (50 mM Tris- $\mathrm{HCl}$ (pH 7.4), 1\% NP-40, 0.25\% Na-deoxycholate, $150 \mathrm{mM} \mathrm{NaCl}, 1 \mathrm{mM}$ EDTA, $1 \mathrm{mM}$ PMSF, $1 \mathrm{mg} / \mathrm{ml}$ aprotinin, $1 \mathrm{mM} \mathrm{Na} \mathrm{VO}_{4}, 1 \mathrm{mM} \mathrm{NaF}$ ) containing a cocktail of protease inhibitors (Calbiochem, Darmstadt, Germany). Cellular protein $(40 \mu \mathrm{g})$ from each sample was applied to $12 \%$ SDS-PAGE gels and probed with specific antibodies. Blots were developed with HRP-conjugated secondary antibodies and chemiluminescence substrate on Kodak X-ray films.

Xenograft mouse model. Animal studies were conducted in full accordance with the Institutional Guide for the Care and Use of Laboratory Animals. All mouse protocols were approved by the Animal Care and Use Committee of Sichuan University (Chengdu, Sichuan, China). Six-week-old female athymic (nu/ $\mathrm{nu}$ ) mice were obtained from the Chinese Academy of Medical Science (Beijing, China). Human tumor xenografts tumors were established by s.c. injection of $5 \times 10^{6}$ cells. Animals were randomized into treatment groups ( $\mathrm{n}=8$ per group) when tumors reached a defined palpable size $\left(100-200 \mathrm{~mm}^{3}\right)$. The dosing schedules were continuous oral administration of TP58 $80 \mathrm{mg} / \mathrm{kg} / \mathrm{day}$ or vehicle for 21 days. Tumor length and width were determined every three days and tumor volume (TV) was calculated using the following formula: $\mathrm{TV}=$ length $\mathrm{x}$ width ${ }^{2} \mathrm{x} 0.52$.

Preparation of cDNA microarray. A human genome 70-mer oligonucleotide microarray was obtained from CapitalBio Corporation (Beijing, China). This microarray contains 35,035 oligonucleotide probes representing approximately 25,100 genes and 39,600 transcripts including control housekeeping genes such as GAPDH, $\beta$-actin and $\alpha$-tubulin. Preparation of cDNA and subsequent steps leading to hybridization, scanning and data analysis were previously described $(16,17)$. In brief, a total of $2 \mu \mathrm{g}$ of the total RNA was labeled and hybridized to $17 \mathrm{~K}$ human microarrays. Fluorescently labeled cDNA was obtained from a single round of labeling using a kit in the presence of fluorescent dNTP (Cy3-dCTP or Cy5-dCTP, GE Healthcare). Hybridization of arrays was preformed in a CapitalBio BioMixer ${ }^{\mathrm{TM}}$ II hybridization station overnight at a rotation speed of $8 \mathrm{rpm}$ and a temperature of $42^{\circ} \mathrm{C}$ and washed in a CapitalBio Slide Washer ${ }^{\mathrm{TM}}$ (CapitalBio) with two consecutive solutions $\left(0.2 \% \mathrm{SDS}, 2 \mathrm{X} \mathrm{SSC}\right.$ at $42^{\circ} \mathrm{C}$ for $5 \mathrm{~min}$, and $0.2 \mathrm{X} \mathrm{SSC}$ for $5 \mathrm{~min}$ at room temperature). Cy3 and Cy5 fluorescence was scanned with a confocal LuxScan ${ }^{\mathrm{TM}}$ scanner and the images obtained were then analyzed using the 

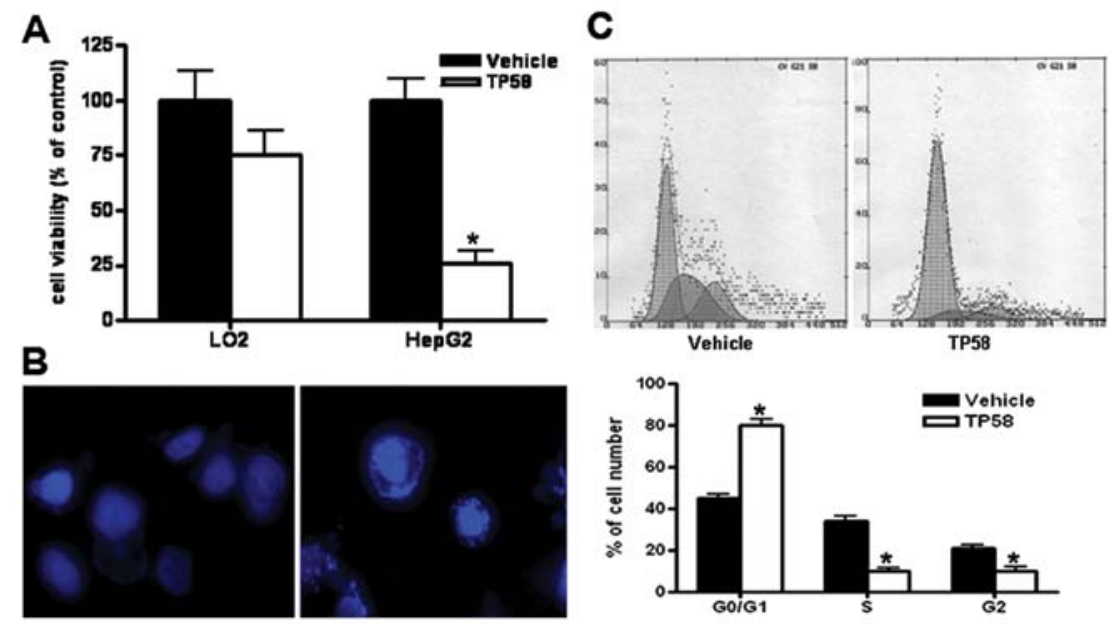

Figure 1. TP58 inhibits HepG2 proliferation, cell cycle progression and promotes apoptosis while exhibiting relatively low cytotoxicity to L02. (A) Proliferation potential of HepG2 and L02 $36 \mathrm{~h}$ after treatment with TP58 or vehicle was determined by the MTT assay. Data are representative of three independent experiments. ("p<0.05, compared to the vehicle-treated counterpart). (B) HepG2 cells were treated with TP58 or vehicle for 36 h, stained with Hoechst 33258 , and observed under a fluorescence microscope. (C) Cell cycle progression of HepG2 $36 \mathrm{~h}$ after treatment with TP58 or vehicle was determined by FACS analysis. A representative of three independent experiments was shown $\left({ }^{*} \mathrm{p}<0.05\right.$, compared with control cells).

LuxScan 3.0 software (both from CapitalBio). To determine the significant differentially expressed genes, Significance Analysis of Microarrays (SAM, version 3.02) were performed.

2-DE (two-dimension) and image analysis. The 2-DE was performed as described previously (18) with minor modifications. Approximately $4 \times 10^{7}$ cells were lysed in lysis buffer (7 M urea, $2 \mathrm{M}$ thiourea, 4\% CHAPS, $100 \mathrm{mM}$ DTT, $0.2 \%$ pH 3-10 ampholyte; Bio-Rad, USA) and sonicated with 5 10 -sec pulses. The homogenate was subjected to centrifugation at $14,000 \mathrm{rpm}$ for $30 \mathrm{~min}$ at $4^{\circ} \mathrm{C}$, and the protein content in the supernatant was determined using a DC protein assay kit with bovine serum album as a standard. IPG strips (17 cm, pH 7-10, non-linear; Bio-Rad) were passively rehydrated using $340 \mu \mathrm{l}$ of rehydration buffer with $2 \mathrm{mg}$ protein for $12-16 \mathrm{~h}$. Isoelectric focusing (IEF) was performed on an IEF cell (Bio-Rad) after equilibration, Electrophoresis of the proteins from strips were carried out using 12\% SDS-PAGE at $30 \mathrm{~mA}$ constant current per gel. After electrophoresis, the gels were stained with Coomassie Brilliant Blue G-250 (Bio-Rad) and imaged using a GS-800 Scanner (Bio-Rad). The protein spots were detected, quantified, and matched using by PDQuest software version 6.1 (Bio-Rad). Statistical analysis was performed by applying the paired t-test. Each sample was run in four replicates.

In-gel digestion. In-gel digestion of proteins was performed using MS-grade Trypsin Gold (Promega, Madison, WI, USA) in accordance with the manufacturer's instructions. Briefly, spots were excised and destained twice with a buffer containing $100 \mathrm{mM} \mathrm{NH} \mathrm{HCO}_{3} / 50 \% \mathrm{ACN}$ at $37^{\circ} \mathrm{C}$ for 45 min each time. After dehydration in ACN for $1 \mathrm{~h}$, the gels mixed with 10-20 $\mu \mathrm{l}$ trypsin solution were incubated for $1 \mathrm{~h}$. Then, a $15 \mu \mathrm{l}$ digestion buffer $\left(40 \mathrm{mM} \mathrm{NH} \mathrm{HCO}_{3} / 10 \% \mathrm{ACN}\right)$ was added to cover each gel and incubated overnight at $37^{\circ} \mathrm{C}$. MilliQ water was used to extract tryptic digests initially, followed by extraction using $50 \% \mathrm{ACN} / 5 \%$ TFA. Extracts with peptides were collected and dried in a vacuum concentrator at room temperature and then subjected to MS analysis.
MS/MS analysis and protein identification. Proteins were identified by either ESI- or MALDI-Q-TOF MS. For ESI-Q-TOF MS identification, mass spectra were acquired using a Q-TOF mass spectrometer (Micromass, Manchester, UK) coupled with ESI ions (Micromass). Tryptic digests $(1 \mu \mathrm{l})$ dissolved in $5 \mu 170 \%$ ACN/0.1\% TFA was mixed with $1 \mu 1$ saturated $\alpha$-cyano-4-hydroxy-cinnamic acid (CHCA) in $50 \%$ ACN/0.5\% TFA, which was plated onto a 96-well target plate. For MS/MS analysis, a data dependent mode was used and the highest of the former 10 ions for each MS scan were selected. The MS/MS data obtained and processed using MassLynx v 4.1 software (Micromass) was transformed to PKL files and the instrument was selected as MALDI-Q-TOF. The PKL files were analyzed by the MASCOT search engine (http://www. matrixscience.com). Database searches were carried out using the following parameters: Database, Swiss-Prot; taxonomy, Homo sapiens; enzyme, trypsin; and allowance of one missed cleavage. Carbamidomethylation was determined as a fixed modification and oxidation of methionine was variable. The peptide and fragment mass tolerance were respectively set at 0.1 and 0.2 Da. Proteins with probability based MOWSE scores exceeding their threshold $(\mathrm{p}<0.05)$ were positively identified.

Statistical analysis. The analyses were performed using SPSS 11.5. Data were analyzed statistically using Student's t-test and one way ANOVA. Differences between the groups were considered significant when $\mathrm{p}<0.05$.

\section{Results}

TP58 with relative low cytotoxicity towards L02 inhibits HepG2 proliferation, cell cycle progression and promotes apoptosis. To assess whether TP58 only targets HCC cells and not normal hepatocellular cells, the MTT assay was performed to evaluate its inhibitory effects on proliferation of HepG2 and L02, which are hepatocellular carcinoma and normal liver cells, respectively. In contrast with relatively low cytotoxicity to L02, treatment of TP58 markedly inhibited the proliferation poten- 
A

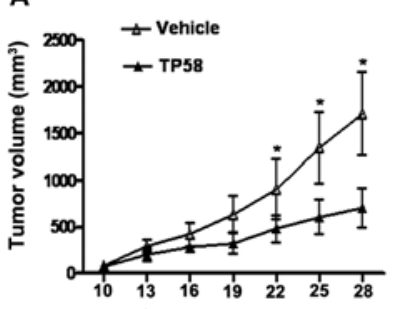

B

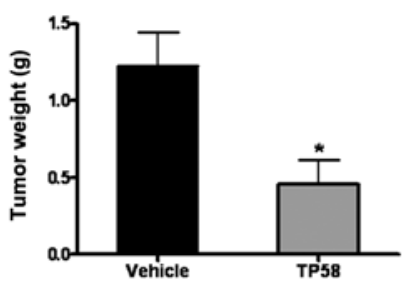

Figure 2. Antitumor effects of TP58 in vivo. (A) HepG2 tumor-bearing nude mice were administered TP58 at $80 \mathrm{mg} / \mathrm{kg}$ or vehicle orally. The treatment with TP58 resulted in significant inhibition of tumor growth vs. the vehicle control. ( $\mathrm{p}<0.01, \mathrm{n}=8$ ). (B) The average weight of HepG2 tumors from the vehicle group were $1.23 \pm 0.49 \mathrm{~g}$, whereas for the TP58 group were $0.55 \pm 0.35$ g. $(\mathrm{p}<0.05, \mathrm{n}=8)$.

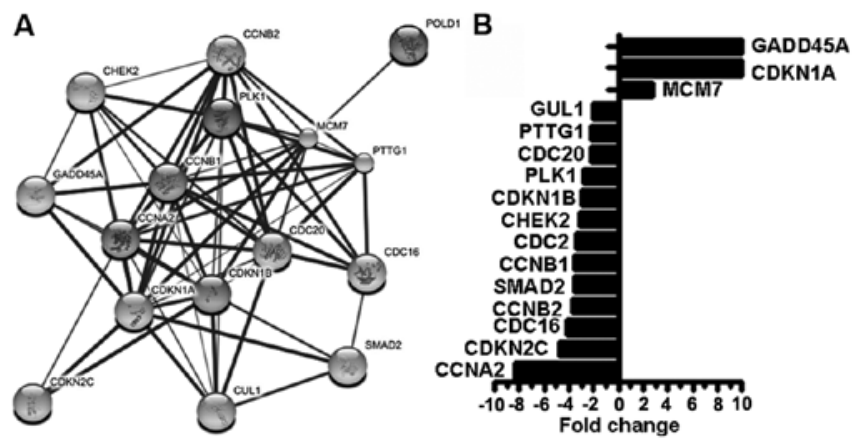

Figure 3. Network analysis of 16 cell cycle associated genes altered by TP58. (A) String 9.0 analysis of 16 changed genes associated with cell cycle regulation pathway following treatment with TP58. (B) Microarray results corresponding to the 16 genes. The relative mRNA expression level was expressed as gene expression levels in the TP58 group compared to the vehicle group.

tial of HepG2 cells (Fig. 1A), suggesting few adverse effects on normal liver tissue. In order to investigate the appearance of apoptosis induced by TP58, Hoechst staining was performed. Compared to normal nuclei appearance without nuclear fragmentation in the control group, cells exposed to TP58 showed abnormal nuclei appearance with nuclear fragmentation indicating appearance of apoptosis (Fig. 1B). Given that cells proliferate via a mitotic process determined by progression through the cell cycle, effects of TP58 on the cell cycle phase distribution were analyzed by flow cytometry. The cell cycle progression of HepG2 cells (Fig. 1C) treated with TP58 was stalled at the G0/G1 phase with a significant decrease in the $S$ and $\mathrm{G} 2 / \mathrm{M}$ phases compared to cell administration of vehicle control. These results indicate that TP58, with relatively low cytotoxicity towards normal liver cell, had a significant antitumor activity in vitro through inhibition of proliferation, cell cycle progression and promotion of apoptosis.

Inhibitory effects of TP58 on the growth of HepG2 xenograft tumors in nude mice. The HepG2 xenograft tumor model was used to compare tumor growth between animals with or without TP58 treatment. Nude mice bearing HepG2 xenografts were treated orally with $80 \mathrm{mg} / \mathrm{kg} /$ day or TP58 vehicle for 21 days. As shown in Fig. 2, TP58 significantly suppressed liver tumor volume and caused more than $60 \%$ inhibition of

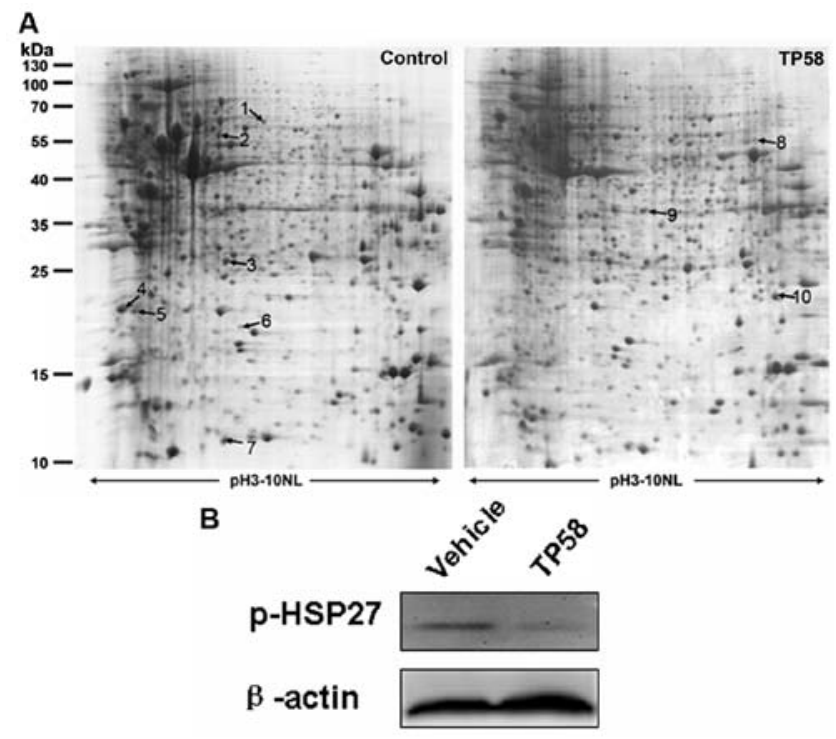

Figure 4. Representative 2-DE gel images and MS/MS analysis. (A) Total protein extracts were separated on $\mathrm{pH} 3-10$ nonlinear IPG strips in the first dimension, followed by $12 \%$ SDS-PAGE in the second dimension and visualized by Coomassie Brilliant Blue staining. In total, 10 differentially expressed spots were identified by MS/MS analysis (marked with arrow and number). Information on each numbered spot was reported in Table I. (B) HepG 2 cells were treated with or vehicle for $36 \mathrm{~h}$, then western blot analysis was performed to determine the protein level of p-HSP27. $\beta$-actin was utilized as a control.

tumor growth compared to vehicle-treated mice. Moreover, the health status of mice treated with TP58 was evaluated. No adverse effects such as weight loss, skin ulcerations or toxic death were observed in the TP58 group.

Cell-cycle regulation by TP58 in HepG2 cells was further confirmed by cDNA microarray analysis. To analyze the effects of TP58 in the gene expression and the cellular process changes in HepG2 cells, cDNA microarray analysis was conducted. Stringent criteria (fold change $\geq 2.0$, up or down) were used to filter the differentially expressed genes. The expression levels of 1174 genes were affected by TP58 (480 upregulated and 694 downregulated) compared to vehicle (data not shown). The gene expression profiles were classified into 902 cellular processes (data not shown) and 156 pathways (data not shown) according to the gene ontology and KEGG. Specifically, cell cycle signaling consisting of 16 differentially expressed genes was the top one pathway affected by TP58. Using String 9.0 analysis, the 16 genes involved in the cell cycle pathway displayed a pattern of complicated gene-gene interactions, as shown in Fig. 3. These findings further confirm the result of the cell cycle block by TP58 in Fig. 2B.

Identification of p-HSP27 as a target of TP58 by two-dimensional analysis. In order to ascertain the liver-specific targets of TP58, proteomics analysis of HepG2 cells treated with TP58 and vehicle was performed. As shown in Fig. 4, 30 protein spots displayed more than 2 -fold changes $(\mathrm{p}<0.05), 9$ of which were successfully identified (Fig. 4A): 4 of the proteins identified were upregulated, whereas 5 were downregulated. The consistently expressed proteins with their expression profile are summarized in Table I. Expression levels of p-HSP27 was 


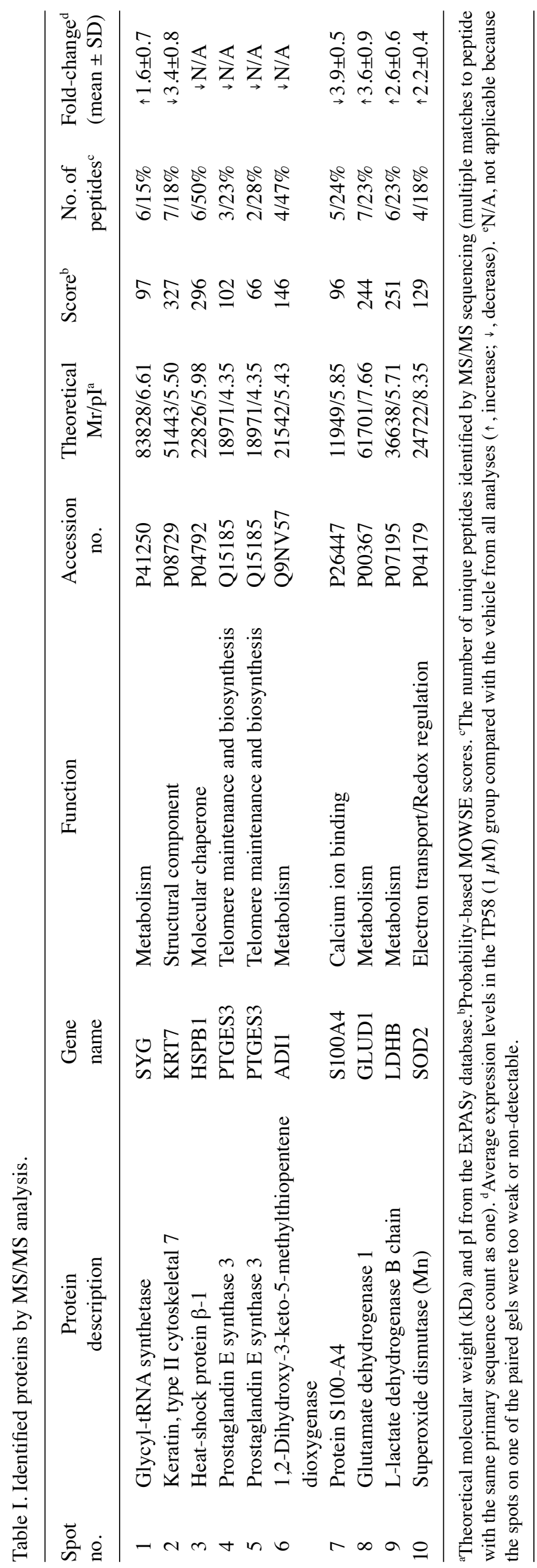

A

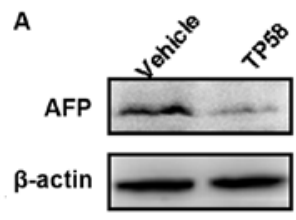

$\beta$-actin

B

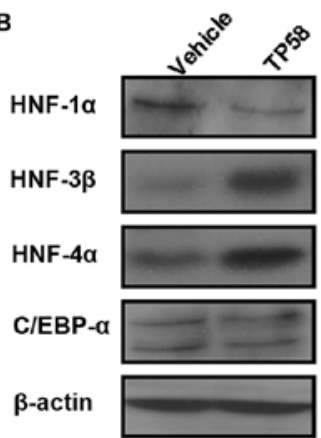

Figure 5. TP58 modulates LETFs and AFP expression in vitro. HepG2 cells were treated with TP58 for $36 \mathrm{~h}$, and expression of AFP (A) and liverenriched transcription factors (LETFs) (B) was determined by western blot analysis. Data are representative of three independent experiments.

further confirmed by western blot analysis given that it not only serves as a biomarker of tumor including HCC (19) but also participates in cell cycle regulation $(20,21)$. The result of western blot analysis (Fig. 4B) showed that administration of TP58 significantly suppressed the phosphorylation of HSP27. Interestingly, genes encoding protein kinases including PRS6KB1 (ribosomal protein S6 kinase $\beta 1$ ), PRKCBP1 (protein kinase C-binding protein 1), PRKC1 (serine/threonine-protein kinase C1), PRKD2 (serine/threonine-protein kinase D2) and MAP3K5 (mitogen-activated protein kinase kinase kinase 5) participating in phosphorylation of HSP were regulated by TP58 at the transcriptional level shown by microarray (data not shown). Therefore TP58 may modulate p-HSP27 through regulating such protein kinases at the transcriptional level.

TP58 may display HCC-specific anticancer activity by regulating liver-specific LETFs. AFP is not only a diagnostic and prognostic marker of HCC (22) but may also reveal the degree of differentiation $(23,24)$. Thus, the levels of AFP was determined by western blot analysis (Fig. 5A). Exposure to TP58 markedly downregulated the AFP expression compared to vehicle suggesting that TP58 may be involved in the regulation of differentiation. Then western blot analysis was conducted to examine levels of LETFs which play an important role in the development and differentiation of liver through relative restricted expression in liver and the regulation of liver-specific genes transcription such as AFP $(13,14)$. Treatment with TP58 significantly upregulated HNF-3 $\beta$ and HNF-4 $\alpha$ but downregulated HNF-1 $\alpha$ (Fig. 5B). C/EBP $\alpha$ did not display an obvious difference between the TP58 and the vehicle group.

\section{Discussion}

Despite progress made in the treatment of $\mathrm{HCC}$, novel therapies are needed. In our previous study, TP58, a novel thienopyridine derivative compound targeting Aurora kinase displayed most potent and relatively specific inhibition of proliferation of the human hepatoma HepG2 cells in vitro, which could not be explained by targeting Aurora kinase. Therefore, it is worthwhile to further study the anticancer effect of TP58 in liver tumor growth and to explore the underlying molecular mechanism therein. 


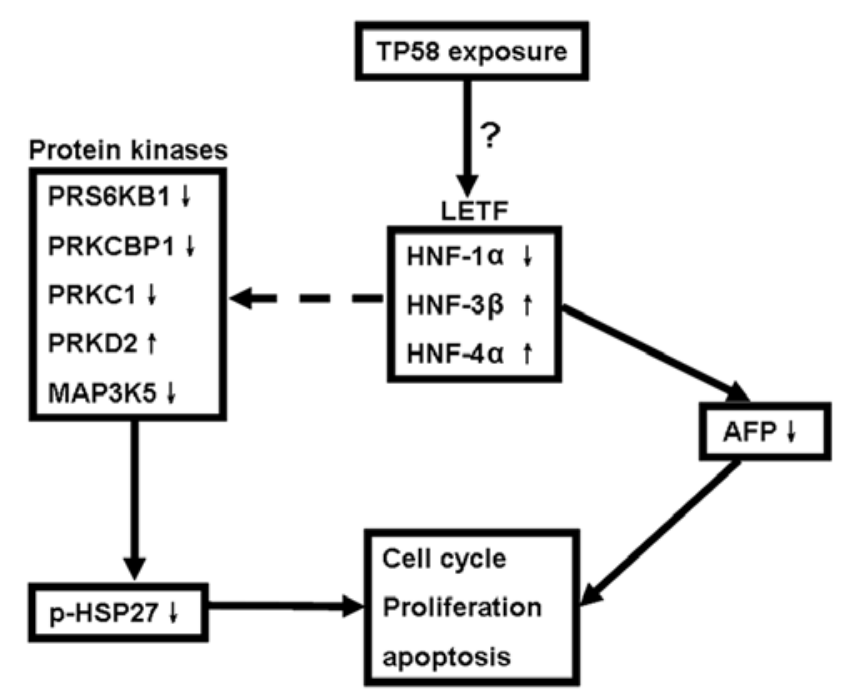

Figure 6. A hypothetical model illustrating the possible signaling pathways by which TP58 inhibits HCC. Through directly or indirectly modulating liver-specific LETFs, TP58 displayed HCC-specific antitumor activity. Genes indicated by ' $\uparrow$ ' represent proteins upregulated and genes by ' $\downarrow$ ' are downregulated following TP58 treatment. Dotted arrows indicate hypothetica interrelations.

In this study, significant effects of TP58 on the inhibition of tumor growth were observed in both in vitro and in vivo studies. In addition to inhibition of proliferation, TP58 treatment resulted in cell cycle arrest in the G0/G1 phase and promotion of apoptosis. Furthermore, remarkable suppression of in situ liver tumor growth by TP58 treatment was displayed longitudinally at different time points in the HepG2 xenograft tumor model.

To explore the underlying molecular mechanism of HCC-specific anticancer activity by TP58, two high throughput methods, cDNA microarray and two-dimensional protein gel electrophoresis technology were then utilized. In line with cell cycle arrest by TP58 in the in vitro study, results from microarray analyses revealed that cell cycle signaling consisting of 16 differentially expressed genes, interacting with each other was the top one pathway affected by TP58. This was also consistent with the role of cell cycle arrest of other Aurora kinase inhibitors $(25,26)$.

Using proteomic methods, p-HSP27 was identified as a critical downstream target of TP58. HSP27 acts as an ATP-independent chaperone in protein folding, but is also implicated in the architecture of the cytoskeleton, cell migration, metabolism, cell survival, growth/differentiation, mRNA stabilization, and tumor progression (27). It was reported that in addition to serving as a biomarker of HCC (19), levels of HSP27 were correlated inversely with HCC patient progression $(20,21)$. As a form of post-transcriptional modification, p-HSP27 enhanced invasion and metastasis of HCC. Data mining of cDNA microarray demonstrated that genes encoding protein kinases including RPS6KB1, PRKCBP1, PRKC1, PRKD2 and MAP3K5 participating in phosphorylation of HSP were regulated by TP58 at least at the transcriptional level, suggesting that TP58 may modulate p-HSP27 through regulating transcription of such protein kinases.

As a diagnostic and prognostic biomarker of HCC, AFP displayed remarkable reduction after TP58 exposure. Members of LETFs were then determined which play an important role in the development and differentiation of the liver through relative restricted expression in the liver and regulation of the transcription of liver-specific genes, such as AFP. Consistent with our hypothesis, HNF-3 $\beta$ and HNF- $4 \alpha$ were significantly upregulated when exposed to TP58.

A diagram of a hypothetical model of the signaling pathways involved in TP58-induced inhibition of HCC is shown in Fig. 6. We hypothesize that p-HSP27 and AFP are the indirect but critical targets of TP58, while regulation of LETFs by TP58 in the upstream pathway supports the notion that the mechanism of HCC-specific antitumor activity by TP58 includes two signaling pathways. The first is the TP58/LETFs/ protein kinases/p-HSP pathway. As a phosphorylated form of HSP27, p-HSP27 contributes to the malignant properties of these cells, including increased tumorigenicity, treatment resistance and apoptosis inhibition (27). By modulating the levels of protein kinases regulating the phosphorylation of HSP27 through LETFs, regardless of the fact that the molecular link between LETFs and protein kinases regulating phosphorylation of HSP27 is less clear, phosphorylation of HSP27 was reduced attenuating the malignant progression of HCC. The second pathway is TP58/ LETFs/AFP. AFP is a diagnostic and prognostic biomarker of HCC. Moreover, recent studies have shown that it also functions as a regulator factor in HCC. By altering the expression of FasL and TRAIL in hepatoma cells and of Fas and TRAILR in lymphocytes, AFP triggers hepatoma cells to escape immune surveillance. Furthermore, AFP has been reported to be a critical determinant in the growth of hepatoma cells via regulation of the cell cycle.

In conclusion, TP58 has shown its great potential in specific suppression of liver tumor growth through downregulation of p-HSP27 and AFP. The HCC-specific antitumor activity is partially dependent on modulation of LETFs. Further research will focus on how TP58 modulates LETFs and will elucidate the molecular link between LETFs and protein kinases regulating phosphorylation of HSP27. This study may lead to the development of novel therapies for liver cancer.

\section{Acknowledgements}

The study was funded by the National Natural Science Foundation of China (no. 81000982) and also supported by the Fundamental Research Funds for the Central Universities (no. SWJTU09BR217 and no. SWJTU09ZT28). The authors sincerely thank Yong Wang from The First Affiliated Hospital of Chongqing Medical University (Chongqing, China) for assistance with manuscript preparation.

\section{References}

1. Jemal A, Siegel R, Ward E, Hao Y, Xu J, Murray T and Thun MJ: Cancer statistics, 2008. CA Cancer J Clin 58: 71-96, 2008.

2. Thomas MB and Zhu AX: Hepatocellular carcinoma: the need for progress. J Clin Oncol 23: 2892-2899, 2005.

3. Simonetti RG, Liberati A, Angiolini C and Pagliaro L: Treatment of hepatocellular carcinoma: a systematic review of randomized controlled trials. Ann Oncol 8: 117-136, 1997.

4. Keen N and Taylor S: Aurora-kinase inhibitors as anticancer agents. Nat Rev Cancer 4: 927-936, 2004.

5. Fu J, Bian M, Jiang Q and Zhang C: Roles of Aurora kinases in mitosis and tumorigenesis. Mol Cancer Res 5: 1-10, 2007. 
6. Jeng YM, Peng SY, Lin CY and Hsu HC: Overexpression and amplification of Aurora-A in hepatocellular carcinoma. Clin Cancer Res 10: 2065-2071, 2004.

7. Tanaka S, Arii S, Yasen M, et al: Aurora kinase B is a predictive factor for the aggressive recurrence of hepatocellular carcinoma after curative hepatectomy. Br J Surg 95: 611-619, 2008.

8. Lin ZZ, Jeng YM, Hu FC, et al: Significance of Aurora B overexpression in hepatocellular carcinoma. Aurora B overexpression in HCC. BMC Cancer 10: 461, 2010.

9. Lin ZZ, Hsu HC, Hsu CH, et al: The Aurora kinase inhibitor VE-465 has anticancer effects in pre-clinical studies of human hepatocellular carcinoma. J Hepatol 50: 518-527, 2009.

10. Benten D, Keller G, Quaas A, et al: Aurora kinase inhibitor PHA-739358 suppresses growth of hepatocellular carcinoma in vitro and in a xenograft mouse model. Neoplasia 11: 934-944, 2009.

11. Aihara A, Tanaka S, Yasen M, et al: The selective Aurora B kinase inhibitor AZD1152 as a novel treatment for hepatocellular carcinoma. J Hepatol 52: 63-71, 2010.

12. Zeng XX, Zheng RL, Zhou T, et al: Novel thienopyridine derivatives as specific anti-hepatocellular carcinoma (HCC) agents: synthesis, preliminary structure-activity relationships, and in vitro biological evaluation. Bioorg Med Chem Lett 20 : 6282-6285, 2010.

13. Cereghini S: Liver-enriched transcription factors and hepatocyte differentiation. FASEB J 10: 267-282, 1996.

14. Hayashi $\mathrm{Y}$, Wang W, Ninomiya T, Nagano $\mathrm{H}$, Ohta $\mathrm{K}$ and Itoh $\mathrm{H}$ : Liver enriched transcription factors and differentiation of hepatocellular carcinoma. Mol Pathol 52: 19-24, 1999.

15. Heo DS, Park JG, Hata K, Day R, Herberman RB and Whiteside TL: Evaluation of tetrazolium-based semiautomatic colorimetric assay for measurement of human antitumor cytotoxicity. Cancer Res 50: 3681-3690, 1990.

16. Patterson TA, Lobenhofer EK, Fulmer-Smentek SB, et al: Performance comparison of one-color and two-color platforms within the Microarray Quality Control (MAQC) project. Nat Biotechnol 24: 1140-1150, 2006.

17. Guo Y, GuoH, Zhang L, et al: Genomic analysis of anti-hepatitis B virus (HBV) activity by small interfering RNA and lamivudine in stable HBV-producing cells. J Virol 79: 14392-14403, 2005.
18. Tong A, Zhang H, Li Z, et al: Proteomic analysis of liver cancer cells treated with suberonylanilide hydroxamic acid. Cancer Chemother Pharmacol 61: 791-802, 2008.

19. Feng JT, Liu YK, Song HY, et al: Heat-shock protein 27: a potential biomarker for hepatocellular carcinoma identified by serum proteome analysis. Proteomics 5: 4581-4588, 2005.

20. Venkatakrishnan CD, Dunsmore K, Wong H, et al: HSP27 regulates p53 transcriptional activity in doxorubicin-treated fibroblasts and cardiac H9c2 cells: p21 upregulation and G2/M phase cell cycle arrest. Am J Physiol Heart Circ Physiol 294: H1736-H1744, 2008.

21. Matsushima-Nishiwaki R, Takai S, Adachi S, et al: Phosphorylated heat shock protein 27 represses growth of hepatocellular carcinoma via inhibition of extracellular signalregulated kinase. J Biol Chem 283: 18852-18860, 2008.

22. Fujiyama S, Tanaka M, Maeda S, Ashihara H, Hirata R and Tomita K: Tumor markers in early diagnosis, follow-up and management of patients with hepatocellular carcinoma. Oncology 62 (Suppl 1): 57-63, 2002.

23. Tangkijvanich $P$, Anukulkarnkusol N, Suwangool $P$, Lertmaharit $S$, Hanvivatvong O, Kullavanijaya $\mathrm{P}$ and Poovorawan Y: Clinical characteristics and prognosis of hepatocellular carcinoma: analysis based on serum alpha-fetoprotein levels. J Clin Gastroenterol 31: 302-308, 2000.

24. Wu W, Yao DF, Yuan YM, et al: Combined serum hepatoma-specific alpha-fetoprotein and circulating alphafetoprotein-mRNA in diagnosis of hepatocellular carcinoma. Hepatobiliary Pancreat Dis Int 5: 538-544, 2006.

25. Jetton N, Rothberg KG, Hubbard JG, Wise J, Li Y, Ball HL and Ruben L: The cell cycle as a therapeutic target against Trypanosoma brucei: hesperadin inhibits Aurora kinase-1 and blocks mitotic progression in bloodstream forms. Mol Microbiol 72: 442-458, 2009

26. Yang G, Chang B, Yang F, et al: Aurora kinase A promotes ovarian tumorigenesis through dysregulation of the cell cycle and suppression of BRCA2. Clin Cancer Res 16: 3171-3181, 2010.

27. Kostenko S and Moens U: Heat shock protein 27 phosphorylation: kinases, phosphatases, functions and pathology. Cell Mol Life Sci 66: 3289-3307, 2009. 